\title{
Mental Health and Substance Use Disorder Co-Morbidities Among Teens in Treatment: SASSI-A3 Correlations in Screening Scores
}

\author{
Nelson Jose Tiburcio*, Scarlett Lynn Baker, Kristin Sue Kimmell \\ The SASSI Institute, Springville, Indiana, USA \\ Email address: \\ nelson@sassi.com (N. J. Tiburcio), scarlett@sassi.com (S. L. Baker), kristin@sassi.com (K. S. Kimmell) \\ ${ }^{*}$ Corresponding author
}

\section{To cite this article:}

Nelson Jose Tiburcio, Scarlett Lynn Baker, Kristin Sue Kimmell. Mental Health and Substance Use Disorder Co-Morbidities Among Teens in Treatment: SASSI-A3 Correlations in Screening Scores. Psychology and Behavioral Sciences. Vol. 10, No. 1, 2021, pp. 10-17.

doi: $10.11648 /$ j.pbs. 20211001.12

Received: December 14, 2020; Accepted: January 6, 2021; Published: January 15, 2021

\begin{abstract}
This article presents an overview of a study investigating the co-occurrence of teen mental health and substance use disorders. These co-morbidities have proven problematic and difficult to treat and/or identify in this population. The data for this study was collected as part of The SASSI Institute's third iteration of the Adolescent Substance Abuse Subtle Screening Inventory (SASSI-A3). A total of 515 teenagers in treatment, whose cases consisted of a valid and complete SASSI-A3, DSM-5 diagnostic evaluation, and client demographics, served as the dataset for the present study. Specifically, we focused on the frequencies of mental health diagnoses alongside a DSM-5 diagnosis of substance use disorder for teens who were seeking treatment for substance misuse issues. All participant cases were provided by clinicians working in service settings throughout the U.S. Census Regions (Northeast, Midwest, South, West). These professionals served in a variety of venues including substance use treatment and criminal justice programs, community corrections, private clinical practices, behavioral health centers, and social service organizations. All clinicians were qualified SASSI users who administered the SASSI-A3 via the SASSI Institute's SUD web-based screening application. Substance abuse in teens can often be a sign of an attempt to self-medicate an untreated mental health disorder. Identifying possible correlations between SASSI-A3 scale scores and diagnosed mental health disorders, depression and anxiety in particular, can enable clinicians to direct the course of subsequent clinical interviews, and further assessments needed early in the counseling relationship.
\end{abstract}

Keywords: Adolescent, Substance Use Disorders, SASSI, Co-occurring SUD-mental Health Disorders

\section{Introduction}

Adolescents who are referred for drug treatment assessments, particularly those who are mandated due to acting out behaviors, are often screened for substance use disorders (SUDs). However, it is sometimes the case that when an adolescent is identified as having an SUD, further assessment for co-occurring mental health disorders is not conducted, unless warranted or ordered (Community court judges, etc.). Generally, this occurs because different types of professionals specialize in the treatment of specific disorders and as some studies have shown historically, might not coordinate behavioral service provision. For example, typically, substance abuse providers conduct a thorough substance misuse assessment, but only a cursory evaluation of mental health issues; conversely, mental health providers complete a thorough mental health evaluation, but a cursory evaluation of substance use issues [1]. More recent data has shown an improvement in parsing out the mental health from substance use issues, but a clear need still remains when examining these issues with this population. For example, Substance Abuse and Mental Services Administration's (SAMHSA) [2] national survey reported that substance use in teens is more common in those with mental health disorders (MHD) than those without an MHD. Recent work by the National Institute on Drug Abuse (NIDA) and other government agencies are helping to ensure that individuals receive integrated treatment to address both issues. In 
addition, identification of any possible co-occurring MHD/SUD as soon as possible in the treatment relationship has demonstrated efficacy in preventing future relapse and added complications [3]. Unfortunately, there still exist significant gaps in our understanding and implementation of effective and efficient service and care provision for people suffering from co-occurring disorders (CODs).

\section{Background and Significance}

First exposure to alcohol and other drugs and the onset of related issues for most individuals occurs during the teenage years, highlighting the importance of early intervention. This adolescent period is also when MHDs (mood, anxiety, etc.) commonly occur in combination with SUD [3, 4]. However, differential diagnosis in teens remains challenging, as symptoms in teens with MHD tend to be nonspecific [5]. That is, the teen presents symptoms characteristic of a disorder that cause clinically significant distress or social, occupational, or other important areas of functioning, but do not meet the full criteria for a specific disorder within a disorder classification group [6]. In these cases, the clinician can choose not to specify the disorder. For example, they might diagnose an unspecified anxiety disorder rather than a specific anxiety disorder such as panic disorder or social anxiety disorder (social phobia).

It is imperative to routinely screen adolescents with a MHD diagnosis for SUD. Substance use by these teens is especially concerning and should be monitored closely due to the potential impact of substance use on the MHD and possible interactions between the substance and any prescribed medication [7]. Additionally, psychiatric comorbidity was shown in a recent study by Lyons, Yule, Schiff, Bagley, \& Wilens [8] to be a noteworthy predictor of overdose in young people. The SASSI Institute engages in research to evaluate the psychometric properties of our various substance use measures and to enhance their accuracy and clinical utility. For the present article, we reviewed clinicians' independent diagnosis of the presence of MHD and their diagnosis for the presence of SUD according to the diagnostic criteria from the DSM-5 [6]. Since the initial release of the SASSI in 1988, professionals using SASSI instruments have reported general clinical observations about correlates on the SASSI screening scale scores, especially in regards to clients with comorbidity. Thus, we decided to analyze the SASSI-A3 validation data to see if it would demonstrate correlations in certain combinations of scale scores in clients diagnosed with MHD and a co-occurring SUD supporting these reported clinical hypotheses.

\section{The Existing Evidence Base on Adolescent Co-occurring Disorders}

Untreated, co-occurring disorders increase risk for self-harm. Mental health disorders and substance use are tightly linked; often, when a mental health disorder goes undiagnosed or untreated, a young person will attempt to self-medicate or self-treat with drugs or alcohol. Left untreated, mental health disorders can pose serious risks for problematic substance use. Similarly, substance use poses a serious risk for developing a mental health disorder in the future. According to SAMHSA data [3], in 2018, the percentage of adolescents who used illicit drugs in the past year was higher among those with a past year major depressive episode (MDE) than it was among those without a past year MDE (32.7 vs. 14.0 percent).

Adolescents who had a past year MDE also were more than twice as likely as adolescents without an MDE to be past year users of marijuana (25.2 vs. 10.5 percent) or almost three times as likely to be misusers of opioids (i.e., heroin users or misusers of prescription pain relievers) (6.1 vs. 2.2 percent), [9]. Among the 358,000 adolescents aged 12 to 17 in 2018 who had a co-occurring MDE and an SUD in the past year, 234,000 received either substance use treatment at a specialty facility or mental health services in the past year. This number of adolescents who received mental health care or specialty substance use treatment corresponds to 65.7 percent of adolescents who had a co-occurring MDE and an SUD. Stated another way, more than a third of adolescents with both an MDE and an SUD in the past year did not receive either type of service. Among adolescents in 2018 with a co-occurring MDE and an SUD, 5.4 percent received both mental health care and specialty substance use treatment, 59.5 percent received only mental health care [9].

SAMHSA further reports that in 2018, about 946,000 adolescents aged 12 to 17 needed substance use treatment in the past year. This number corresponds to 3.8 percent of adolescents, or about 1 in 26 adolescents [9]. Concurrently in 2018, approximately 3.7 million people aged 12 or older received any substance use treatment in the past year, or 1.4 percent of the population. Approximately 2.4 million people aged 12 or older in 2018 received substance use treatment at a specialty facility in the past year, or 0.9 percent of the population. During that same year, approximately 83,000 adolescents aged 12 to 17 received substance use treatment at a specialty facility in the past year, which corresponds to 0.3 percent of adolescents.

Moreover, the 83,000 adolescents aged 12 to 17 in 2018 who received substance use treatment at a specialty facility in the past year correspond to 8.8 percent of the adolescents who needed substance use treatment. These percentages of people who received substance use treatment at a specialty facility in 2018, concorded similarly with percentages from 2015 to 2017. During that timeframe, approximately 686,000 people aged 12 or older with an alcohol use disorder received alcohol use treatment at a specialty facility in the past year, or 4.6 percent of people with an alcohol use disorder [3], And again, among people aged 12 or older with an alcohol use disorder, the percentage in 2018 who received alcohol use treatment at a specialty facility was significantly comparable to the percentages in 2015 to 2017.

As regards to receipt of illicit Substance use disorder treatment at a specialty facility among "people with specific illicit substance use disorders (SUD's)," in 2018, approximately 743,000 people aged 12 or older with an SUD received illicit 
drug use treatment at a specialty facility in the past year [9]. This number corresponds to 9.2 percent of people aged 12 or older with an illicit drug use disorder. Among people aged 12 or older with such disorders, these 2018 percentage figures include any Illicit Drug Use Disorder (9.2), Marijuana (5.1), Pain Relievers (15.6), Cocaine (19.0), Opioid Use (19.7) and Methamphetamine Use Disorder (18.1), [9].

\section{Participants}

All participant cases were provided by clinicians working in service settings throughout the U.S. Census Regions (Northeast, Midwest, South, West). These professionals served in a variety of venues including substance use treatment and criminal justice programs, community corrections, private clinical practices, behavioral health centers, and social service organizations. All clinicians were qualified SASSI users who administered the SASSI-A3 via the SASSI Institute's SUD web-based screening application. In appreciation for the use of their anonymous responses, the SASSI Institute made a \$5 donation to the teen's choice of a youth or pet charity.

Table 1. Participant characteristics.

\begin{tabular}{|c|c|}
\hline Characteristic & $(n=515) \%$ \\
\hline \multicolumn{2}{|l|}{ Data Source } \\
\hline Criminal Justice Programs & 3.50 \\
\hline Social Services Programs & 4.66 \\
\hline Substance Use Treatment & 82.33 \\
\hline Other & 9.51 \\
\hline \multicolumn{2}{|l|}{ Clinical Diagnosis } \\
\hline Mild Substance Use Disorder & 17.67 \\
\hline Moderate Substance Use Disorder & 14.56 \\
\hline Severe Substance Use Disorder & 34.76 \\
\hline Criterion Negative & 33.01 \\
\hline \multicolumn{2}{|l|}{ Gender } \\
\hline Male & 65.44 \\
\hline Female & 34.56 \\
\hline \multicolumn{2}{|l|}{ Employment Status } \\
\hline Employed/Full-time & 1.17 \\
\hline Employed/Part-time & 12.82 \\
\hline Not employed & 84.66 \\
\hline Volunteer & 1.36 \\
\hline \multicolumn{2}{|l|}{ Race/Ethnicity } \\
\hline Black or African American & 17.28 \\
\hline American Indian or Alaska Native & 0.97 \\
\hline Asian, Hawaiian or Pacific Islander & 0.97 \\
\hline Hispanic & 29.90 \\
\hline White or Caucasian & 43.69 \\
\hline Multiracial & 5.83 \\
\hline Other/Unknown & 1.36 \\
\hline \multicolumn{2}{|l|}{ Living Situation } \\
\hline Parents & 60.39 \\
\hline Other Relatives & 7.77 \\
\hline Friends & 0.39 \\
\hline Group Home & 1.75 \\
\hline Residential & 15.53 \\
\hline Other/Unknown & 14.17 \\
\hline \multicolumn{2}{|l|}{ Education (years) } \\
\hline$M$ & 8.54 \\
\hline$S D$ & 1.57 \\
\hline \multicolumn{2}{|l|}{ Age (years) } \\
\hline$M$ & 15.41 \\
\hline$S D$ & 1.42 \\
\hline
\end{tabular}

A total of 580 SASSI-A3 administrations were collected. The responses were evaluated to ensure that missing responses would not affect profile validity. Those with significant missing responses, as well as those cases without sufficient diagnostic information, were excluded from analyses $(n=65)$. A total of 515 teenagers in treatment for a variety of reasons (i.e., alcohol and drug use, suicidal ideation, trauma), whose cases consisted of a valid and complete SASSI-A3, DSM-5 diagnostic evaluation, and client demographics, served as the dataset. The dataset consisted of teens between the ages of 13 and 18 (mean $=15$ years old). Sixty-five percent were Male, thirty-five percent Female. Teens in this group identified themselves as mostly white (44\%), not employed (85\%), and living with parents $(60 \%)$. Clinicians reported that the majority of the teens $(67 \%)$ were diagnosed with an SUD and the remaining 33\% did not have an SUD (DSM-5 criterion negative). Reported severity levels were diagnosed according to the DSM-5. Shown in Table 1 are the client demographic characteristics.

\subsection{Human Rights Protections and HIPAA Adherence}

Overall Risk/Benefit Assessment: This project entailed minimal risk to participants in that study participation consisted of providing anonymous responses on a screening survey regarding alcohol and drug-related experiences and attitudes. The risk of harm is thus no greater than would be encountered in standard psychological testing. In addition, treatment participants were invited to participate in the study by assessment professionals who use the SASSI screening survey in their practices and who have an established professional relationship with the respondent. Clinical licensure, certification, and code of ethics require that counselors consent their clients for treatment with full disclosure of expectations and rights in the client-counselor relationship, including the counselor's agreement to act in the best interest of the client. Further, both parents and teens decided whether to provide permission and assent to study participation. As in other behavioral survey research, participants may, on occasion, feel uncomfortable answering some of the survey questions. But as further protection and to mitigate against these risks, we gave participants the option of skipping any question/s or withdrawing from study participation at any time, without incurring any penalty or rescinding any rights to which they would otherwise be entitled.

\subsection{Data Collection Procedures}

We facilitated a separate research module on the SASSIOnline platform in order to allow participating counselors to administer the research survey to responding adolescents. We encrypted all data transmissions and de-identified client information so that all identifiable client information was maintained as encrypted data. Parental consent and teen assent were verbally obtained prior to participation by their counselor. Respondents were also given the option to discontinue their participation at 
any time or have their completed survey withdrawn from the study by informing their counselor. The Advarra Institutional Review Board (IRB) reviewed and approved all protocols prior to study commencement to ensure that participants were treated in accordance with HIPAA guidelines and regulations.

Additional procedural and more elaborate methodological discussions on the development and validation of the adolescent SASSI-A3 substance use disorder screening inventory are discussed in Tiburcio, Baker, \& Hanauer [10] and Tiburcio, Hanauer, \& Baker [11].

\section{Measures}

\subsection{Research Version of the SASSI-A3}

The research version of the SASSI-A3 utilized in this study consists of 87 true-false items and 24 face valid alcohol and other drug frequency items that measure how often $(0=$ never to 3 = repeatedly) respondents have engaged in and experienced effects from the use of alcohol and other drugs within a specified time frame. The items on the instrument consist of face valid and subtle items. Face valid items are direct/obvious questions about substance use. Subtle items are questions that make no obvious reference to substance use but are effective in identifying individuals likely to have an SUD, regardless of whether or not they are able or willing to acknowledge substance use behaviors [12]. These items are organized into nine scales that are utilized in a series of decision rules to produce a dichotomous SUD screening classification. There are two possible outcomes: "high probability" or "low probability" of SUD. Presently, we are solely focusing on the SASSI scale score correlations and not the SASSI-A3 screening outcome.

The nine scales consist of the following: FVA (Face-Valid Alcohol) - acknowledged consequences of alcohol use, motivation and consequences of usage; FVOD (Face-Valid Other Drugs) - acknowledged consequences of other drug use, motivation and consequences of usage; FRISK (Family and Friends Risk) - part of a family/social system that is likely to enable substance misuse; ATT (Attitudes toward Substance Use) - attitudes and beliefs regarding substance use; SYM (Symptoms of Substance Misuse) - consequences of substance misuse; OAT (Obvious Attributes) - extent to which the individual is aware of and able and willing to acknowledge, some behavioral characteristics that may accompany substance misuse; SAT (Subtle Attributes) helps identify individuals who lack awareness and insight into the nature of their substance misuse and other related problems they may have; DEF (Defensiveness) - identifies defensive responding and lack of forthright disclosure not necessarily related to substance use; and SAM (Supplemental Addiction Measure) - supplements other scales in some decision rules.

An additional scale, VAL (Validity Check), is an empirically derived scale that can be used to identify test negative cases (i.e., low probability of having an SUD on the instrument) that are relatively likely to be false negatives (i.e., adolescents who are incorrectly classified as having a low probability of having an SUD). This helps identify some individuals for whom further evaluation may be of value due to an increased chance of a test miss. The SASSI screening tool also includes a COR (Correctional) scale, but is not used in test classification. The COR scale provides information pertaining to the possibility that the individual may have a relatively high risk of experiencing legal problems.

\subsection{DSM-5}

Clinicians' diagnoses regarding the presence or absence of substance use disorders were obtained in accordance with the American Psychiatric Association's DSM-5 symptom criteria [6]. Counselors indicated the presence or absence SUD symptoms, and specified for what class of drug the symptom was evidenced within the time period for which they conducted each diagnostic evaluation. The Clinician completed the DSM-5 checklist prior to having access to the client's SASSI-A3 screening results. Additionally, at the time the DSM-5 checklist was completed, the clinician was instructed to indicate any co-occurring MHDs that the clinician also diagnosed present for the client.

\section{Data Analysis and Results}

Data analyses were conducted using the SPSS statistical package (Version 22). Of the 515 teenagers in our sample, $40 \%$ were identified by their counselor as having a co-occurring MHD with SUD, and 27\% were identified as only having an SUD diagnosis. Eight percent of the participating teens had a non-co-occurring MHD, and $25 \%$ had no diagnosis. Among the $40 \%(n=205)$ identified as having co-occurring SUD/MHD diagnoses, sixty-two percent were identified as suffering from depression, 49\% anxiety, 16\% Post Traumatic Stress Disorder (PTSD), 36\% Attention-Deficit/Hyperactivity Disorder (ADHD), and 28\% with other MHDs (such as Bipolar, and increasingly prevalent and debilitating Eating disorders). Of particular significance, is that a total of $90 \%$ of the clients were diagnosed with more than one co-occurring MHD.

We also took into account "drug of choice" among those with co-occurring disorders. Marijuana SUD diagnosis was significantly higher among all MHDs combined at 91\%, compared to alcohol at $25 \%$. This is possibly attributable to adolescent beliefs that marijuana use is safer than alcohol, and/or the use of other drugs, with many stating that it is "not harmful because it occurs naturally" [13]. Table 2 presents the breakdown of drug of choice among various MHD disorders.

We also examined teens' scale scores on previously identified "flags for further evaluation of an MHD" on the adolescent SASSI, in relation to a diagnosis of a co-occurring MHD along with SUD [14]. Of the teens with a co-occurring disorder $(n=205), 86 \%$ had elevated COR scores (above the 85 th percentile), and $63 \%$ had low DEF scores (below the 15 th percentile). Table 3 shows the scale scores' percentage amongst the various MHDs. 
Table 2. Co-occurring Mental Health Disorders and SUD diagnosis by drug of choice.

\begin{tabular}{|c|c|c|c|c|c|}
\hline Characteristic SUD Diagnosis & Depression (n-127) \% & Anxiety $(n=101) \%$ & PTSD $(n=32) \%$ & ADHD $(n=73) \%$ & Other* MHD (n = 58) \% \\
\hline Alcohol & 28.3 & 24.8 & 37.5 & 19.2 & 46.6 \\
\hline Marijuana & 91.3 & 88.1 & 96.9 & 95.9 & 94.8 \\
\hline Hallucinogens & 7.1 & 7.9 & 6.3 & 6.8 & 8.6 \\
\hline Inhalants & 2.4 & 3.0 & 0.0 & 5.5 & 6.9 \\
\hline Opioids & 3.9 & 5.0 & 9.4 & 2.7 & 12.1 \\
\hline Sedatives & 9.4 & 10.9 & 9.4 & 11.0 & 10.3 \\
\hline Stimulants & 10.2 & 12.9 & 9.4 & 11.0 & 15.5 \\
\hline Other & 10.2 & 11.9 & 15.6 & 6.8 & 15.5 \\
\hline
\end{tabular}

*Includes Feeding and Eating Disorders, Bipolar and related disorders, and other specified and unspecified mental health disorders.

Table 3. SASSI scores for individuals with Mental Health Disorders with co-occurring SUD.

\begin{tabular}{|c|c|c|c|c|c|}
\hline Characteristic SASSI-A3 Scale Score & $\begin{array}{l}\text { Depression } \\
\text { (n-127) \% }\end{array}$ & $\begin{array}{l}\text { Anxiety } \\
(n=101) \%\end{array}$ & $\begin{array}{l}\text { PTSD } \\
(n=32) \%\end{array}$ & $\begin{array}{l}\text { ADHD } \\
(n=73) \%\end{array}$ & $\begin{array}{l}\text { Other* MHD } \\
(\mathrm{n}=58) \%\end{array}$ \\
\hline Low DEF & 70.1 & 70.3 & 78.2 & 56.2 & 63.8 \\
\hline High Face Valid (alcohol \& other drugs) & 74.0 & 69.3 & 81.3 & 68.5 & 74.1 \\
\hline High OAT & 70.0 & 73.3 & 68.9 & 60.3 & 79.2 \\
\hline High COR & 88.9 & 84.3 & 97.0 & 84.8 & 94.8 \\
\hline
\end{tabular}

*Includes Feeding and Eating Disorders, Bipolar and related disorders, and other specified and unspecified mental health disorders.

We conducted Correlation analyses to identify significant SASSI-A3 profile configurations of scale score combinations. We found relationships between high FVA and low DEF, high FVOD and low DEF, high OAT scores and low DEF scores, as well high COR and low DEF among those with any MHD and co-occurring SUD as shown in Table 4. Only PTSD demonstrated a low correlation between High FVOD and Low DEF and High COR and Low DEF. This could likely be due to the low number of cases diagnosed with PTSD in the sample.

Table 4. SASSI scale score correlations among teens with SUD and a co-occurring MHD

\begin{tabular}{|c|c|c|c|c|c|c|}
\hline $\begin{array}{l}\text { Characteristic SASSI-A3 Scale Score } \\
\text { Correlations }\end{array}$ & $\begin{array}{l}\text { Any MHD } \\
(\mathrm{n}=\mathbf{2 0 5})\end{array}$ & $\begin{array}{l}\text { Depression } \\
(n=127)\end{array}$ & $\begin{array}{l}\text { Anxiety } \\
(n=101)\end{array}$ & $\begin{array}{l}\text { PTSD } \\
(n=32)\end{array}$ & $\begin{array}{l}\text { ADHD } \\
(n=73)\end{array}$ & $\begin{array}{l}\text { Other* MHD } \\
(\mathrm{n}=\mathbf{5 8})\end{array}$ \\
\hline High FVA and Low DEF & $-.47 * *$ & $-.45 * *$ & $-.50 * *$ & $-.41 * * *$ & $-.46^{* *}$ & $-.40 * *$ \\
\hline High FVOD and Low DEF & $-.49 * *$ & $-.43 * *$ & $-.55 * *$ & -.18 & $-.58 * *$ & $-.47 * *$ \\
\hline High OAT and Low DEF & $-.70 * *$ & $-.71 * *$ & $-.77 * *$ & $-.70 * *$ & $-.72 * *$ & $-.84 * *$ \\
\hline High COR and Low DEF & $-.50 * *$ & $-.47 * *$ & $-.61 * *$ & -.34 & $-.53 * *$ & $-.56 * *$ \\
\hline
\end{tabular}

*Includes Feeding and Eating Disorders, Bipolar and related disorders, and other specified and unspecified mental health disorders.

$* * \mathrm{p}<.01$.

$* * * \mathrm{p}<.05$.

Gender was also considered when looking at scale score correlations among those with SUD and a co-occurring MHD to see if they were affected by gender. Table 5 reveals no significant differences in correlations as a function of gender.

Table 5. SASSI scale score correlations among teens with SUD and any co-occurring MHD as a function of respondent gender.

\begin{tabular}{lll}
\hline Characteristic SASSI-A3 Scale Score Correlations & Males (n-122) & Females $(\mathbf{n}=\mathbf{8 3})$ \\
\hline High FVA and Low DEF & $-.46^{*}$ & $-.46^{*}$ \\
High FVOD and Low DEF & $-.52^{*}$ & $-.48^{*}$ \\
High OAT and Low DEF & $-.68^{*}$ & $-.72^{*}$ \\
High COR and Low DEF & $-.53^{*}$ & $-.51^{*}$ \\
\hline
\end{tabular}

$* \mathrm{p}<.01$

Given that cultural factors are known to have an impact on an individual's substance use patterns and mental health challenges due to stigma, evaluating whether ethnic group membership affected the correlation of scale scores is of value Table 6 reveals no significant differences between white and non-white minorities.

Table 6. SASSI scale score correlations among teens with SUD and any co-occurring MHD as a function of respondent ethnicity.

\begin{tabular}{lll}
\hline Characteristic SASSI-A3 Scale Score Correlations & White or Caucasian (n-103) & Non-White Minorities $(\mathbf{n}=\mathbf{1 0 2})$ \\
\hline High FVA and Low DEF & $-.44^{*}$ & $-.50^{*}$ \\
High FVOD and Low DEF & $-.50^{*}$ & $-.49^{*}$ \\
High OAT and Low DEF & $-.73^{*}$ & $-.68^{*}$ \\
High COR and Low DEF & $-.59^{*}$ & $-.43^{*}$ \\
\hline
\end{tabular}


Although the sample consisted mostly of white males, when combined, the non-white ethnicities made up $66 \%$ of the sample and $50 \%$ of the teens in the sample who had co-occurring MHD and SUD. Research into treatment for SUD and co-occurring disorders in ethnic/racial minorities has shown that non-white adolescents report receiving lower rates of specialty and informal care, and less informal service [15]. Females made up $35 \%$ of the sample and $40 \%$ of the teens in the sample who had co-occurring MHD and SUD. A study by Latimer, Stone, Voight, Winters, \& August [16] showed that adolescent females exhibit a higher rate of major depression compared to drug-abusing males and are more likely to have co-occurring internalizing problems (e.g., depression and anxiety). The prevalence of these special populations in our research may aid counselors in identifying possible co-occurring SUD and MHD when administering the SASSI-A3, thus increasing the opportunity to access more formal and specialty care for these groups.

\section{Scale Score Interpretations}

Because teens often present in a counselor's office due to legal issues, they are most often screened for SUDs. The SASSI-A3 is a sensitive standardized screening instrument that helps clinicians identify teens who are likely to have an SUD. The instrument can also be informative beyond its use as a screener to aid in treatment planning through the interpretation of scale scores. The DEF scale, and the DEF scale when considered in combination with the FVA, FVOD, OAT, and COR scales provides valuable insight into the clients' behaviors, thus flagging them for further screening for a MHD.

Teens with a low score on the DEF scale (4 or less), have endorsed multiple statements that can be perceived as reflecting negatively on them. This may indicate that they have a tendency toward self-deprecation, which could well be symptomatic of remorse, low self-esteem, and/or a depressed affect. Extreme emotional pain and low self-esteem may be situational, or it may reflect an endogenous trait. Depending on the presenting problems, it may be advisable to do a more comprehensive assessment to look into their possible feelings of hopelessness, suicidal risk, clinical depression, or other psychiatric concerns. Regardless of the cause, if treatment for addictions is to be effective, issues related to low self-esteem or sadness, or both, need to be addressed. Teens in our study who had depression diagnoses had significantly lower DEF scores $(M=3.4)$ than teens without such diagnoses $(M=5.9)$. This empirical evidence suggests that it is worthwhile to further investigate possible depression in teens who have low DEF scores. When a low DEF score presents with high scores on the Face Valid Scales (FVA, and/or FVOD), OAT, or COR scales (above a T-Score of 60), it can add additional insight to the existing interpretation of a low DEF score alone. However, because of the low DEF, it is advisable to screen the client for underlying psychiatric issues.

An elevated score on either the FVA and/or FVOD scales indicates that the teen has no problem identifying themselves as a heavy alcohol and/or drug user. However, if their DEF score is low, coupled with this high face valid score, it indicates they likely have a sense of guilt and fear along with negative self-statements. These scores in combination suggest that the teen may be in crisis and may require immediate care, including assessment to rule out the need for supervised detoxification. Further evaluation may reveal that highly intensive, structured treatment may be necessary to provide adequate support for the teen to initiate and sustain recovery. In this case, they are likely to respond well to supportive group programming, including appropriate self-help groups. This may be particularly important in helping the teen improve her/his self-esteem and self-image. Consistent efforts will likely be necessary to help the teen increase insight and self-awareness regarding the nature of their substance use problems and the way in which these have pervaded their life. They will need considerable support, structure, and supervision in making difficult changes necessary to avoid continued substance misuse.

Elevated OAT scores in combination with low DEF scores indicate that the teen is self-deprecating and may have difficulty using any feedback given to them constructively. While a high OAT indicates that the teen seems to be aware of problematic behaviors related to their substance misuse, clinicians should not expect this to mean that the teen will have the necessary insight to understand the cause of their problems or that they will be willing to change problematic behavior. However, they may be likely to identify with other teens who are working to overcome problems related to their substance misuse, which may increase their insight into the causes of their problems and provide the support and encouragement necessary to make behavioral changes.

If the teen has an elevated COR score, they are at risk of acting out behavior. If they have an existing legal history, it is worth assessing the degree of risk for ongoing problems in the criminal justice system. When combined with a low DEF score, they are likely experiencing a combination of emotional pain and impulsiveness. Clinical assessment may likely reveal anger management issues, poor impulse control, lack of social skills, etc. If this is the case, the initial task is to help such teens realize the relationship between their own behavior and some of the consequences they may have experienced. Further evaluation to determine if the teens' behavior is because of their own pathology or dysfunction, possibly related to an undiagnosed MHD, or if it is in response to a situational crisis or stress, is strongly recommended (See also, Kimmell [17] and Tiburcio, Baker, \& Kimmell [18] for more detailed descriptions and interpretations of SASSI-A3 Scale Scores).

\section{Discussion}

Depression and anxiety were the most identified MHDs to co-occur with SUD amongst our sample group. This is consistent with recent reports that rates of anxiety, depression, and suicidal thoughts are on the rise among teens [19]. 
Teenagers with psychiatric conditions are at higher risk of abusing drugs than other youth and early drug abuse may increase the risk of, or accelerate the course of, psychiatric disorders $[5,20]$.

The most identified SUD among the teens with co-occurring MHD in the sample was marijuana. It has been reported [21] that youths with more mental health symptoms may be using marijuana to cope with underlying symptomatology and thus they may experience more severe social and personal consequences from use when compared with adolescents using only socially or experimentally. Not surprisingly, $97 \%$ of the teens in our sample, reported having been in trouble with the law. With the recent decline in the stigma surrounding marijuana use since the beginning of the 21 st century, teens are more comfortable using marijuana in an effort to work through depression and anxiety on their own. Early identification and treatment of co-occurring disorders in this population is imperative, as studies have also linked chronic cannabis use in teens to an increased risk of schizophrenia [22].

\section{Limitations}

The SASSI-A3 instrument used to collect this data, is an alcohol and drug screening instrument for use as a pre-diagnostic tool as an added source of information during the initial SUD assessment process. The instrument's primary use is to identify teens in need of further evaluation for the presence of an SUD and the potential need for treatment. It is not designed to identify psychiatric disorders or comorbidity with SUDs. The data used for this study was comprised of a convenience sample we intentionally extracted from the larger validation study, and solely for the intent we outlined in the Introduction section. In future studies, we plan to increase the sample size, and conduct data collection to include teens seeking treatment at behavioral health facilities, in addition to SUD treatment settings.

Data used to validate the screening instrument were submitted by practitioners engaged in ongoing programs of substance use assessments and screening with adolescents. Pursuant to IRB regulations and mandates, incarcerated teens, or those in Foster Care were not included in this study. Research including these settings would extend the generalizability of current findings to these populations. In particular, this would allow further investigation into the SASSI-A3 COR scale correlations with low DEF scores in teens being detained in juvenile detention facilities, and identified as having mental health issues, along with SUD. It is also important to note that correlation in scale scores on the SASSI-A3 may not imply causation in all cases. Interpretation of scale scores are hypotheses to explore based on 30 years of professional clinical observations.

\section{Conclusions}

Identifying SUD and/or MHDs in teens is very challenging, especially when they co-occur. Intensely felt experiences and rapid mood shifts are common in adolescence. For example, irritability could indicate depression, bipolar disorder, ADHD, conduct disorder, borderline character disorder, substance abuse withdrawal, or simply be a normal response to the stresses of adolescent development $[4,5]$. Clinicians are challenged with rapidly changing states of openness, compliance, and defiance making it difficult to distinguish if these behaviors are related to substance misuse and/or a psychiatric condition, or just a typical teen acting out.

The co-occurrence of MHDs with SUD appears to be quite prevalent in this sample of teens in treatment. This high prevalence rate of comorbidity supports the need to include a screening tool such as the SASSI for SUD in standard intake and assessment protocols for teens in treatment for psychiatric disorders [23]. Studies show that ADHD, anxiety disorders, posttraumatic stress disorder and depression increase risk of substance use in adolescents. $30 \%-45 \%$ of adolescents and young adults with mental health disorders have a co-occurring substance use disorder, and $65 \%$ or more of youth with substance use disorders also have a mental health disorder [24]. Validated and reliable screening measures, such as the SASSI-A3, enhance access to evidence-based treatments, with added sensitivity to further facilitate access to mental health assessment. Integrated and concurrent treatment of co-occurring disorders within the same clinical spaces and with the same providers is also associated with improved outcomes [25]. Chi, Sterling, Campbell, \& Weisner [26], suggested that twelve-step groups may also be beneficial for addressing adolescent substance use problems, even among those with complex psychiatric disorders and treatment needs. Co-morbid adolescents in that study had comparable or better substance use outcomes than their counterparts over time. It certainly appears that the evidence base for similar studies looking at correlations of SASSI scale scores in such individuals' is clearly at hand.

\section{Acknowledgements}

We wish to thank all the agencies who made valuable contributions to the SASSI-A3 validation study and provided dual diagnosis information for this part of the research project.

\section{References}

[1] Brems, C. Johnson, M. E., and Namyniuk, L. L. (2002) Clients with substance abuse and mental health concerns: A guide for conducting intake interviews. Journal Behavioral Health Services \& Research, 29 (3), 327-334.

[2] Substance Abuse and Mental Health Services Administration. (2020). Substance Use Disorder Treatment for People with Co-Occurring Disorders (Treatment Improvement Protocol (TIP) Series, No. 42, SAMHSA Publication No. PEP20-02-01-004).

[3] Substance Abuse and Mental Health Services Administration. (2018). National Survey of Drug Use and Health. 
[4] Wilson, J. J. (2010). Substance abuse during adolescence. In E. V. Nunes, J. Selzer, P. Levounis, and C. A. Davies, (Eds.), Substance dependence and co-occurring psychiatric disorder: Best practices for diagnosis and clinical treatment (pp. 15-1 15-22). Civic Research Institute.

[5] National Institute on Drug Abuse. (2020). Part 1: The Connection Between Substance Use Disorders and Mental Illness.

[6] American Psychiatric Association. (2013). Diagnostic and statistical manual of mental disorders (5th ed.).

[7] Yule, A. M., and Wilens, T. E. (2015). Substance use disorders in adolescents with psychiatric comorbidity: When to screen and how to treat. Current Psychiatry, 14 (4), 36-39, 47-51.

[8] Lyons, R. M., Yule, A. M., Schiff, D., Bagley, S. M., and Wilens, T. E. (2019). Risk Factors for drug overdose in young people: A systematic review of the literature. Journal of Child Adolescent Psychopharmacology, 29 (7), 487-497.

[9] Substance Abuse and Mental Health Services Administration. (2019). Key substance use and mental health indicators in the United States: Results from the 2018 National Survey on Drug Use and Health (HHS Publication No. PEP19-5068, NSDUH Series H-54).

[10] Tiburcio, N. J., Baker, S. L., and Hanauer, M. (2019). Detecting "faking good" with the adolescent Substance Abuse Subtle Screening Inventory-SASSI-A3: A clinical response to alcohol $\&$ other drug use minimization among teens. Alcoholism Treatment Quarterly, 38 (3), 356-363.

[11] Tiburcio, N. J., Hanauer, M., and Baker, S. L. (2020). Validation of the Adolescent Substance Abuse Subtle Screening Inventory-3 (SASSI-A3). Journal of Addiction Research \& Therapy. 11 (5), 400.

[12] Laux, J. M., Piazza, N. J., Salyers, K., and Roseman, C. P. (2012). The Substance Abuse Subtle Screening Inventory-3 and stages of change: A screening validity study. Journal of Addictions \& Offender Counseling, 33, 82-92.

[13] American Academy of Child and Adolescent Psychiatry. (2019). Marijuana and teens.

[14] Lazowski, L. E, and Miller G. A. (2013). The Updated SASSI-A2 User's Guide. The SASSI Institute.

[15] Alegria, M., Carson, N. J., Goncalves, M., and Keefe, K. (2012). Disparities in treatment for substance use disorders and co-occurring disorders for ethnic/racial minority youth. Journal of the American Academy of Child \& Adolescent Psychiatry, 50 (1), 22-31.

[16] Latimer, W. W., Stone, A. L., Voight, A., Winters, K. C., and August, G. J. (2002). Gender differences in psychiatric comorbidity among adolescents with substance use disorders. Experimental and Clinical Psychopharmacology, 10 (3), 310-315.

[17] Kimmell, K. S. (2020). SASSI Scales: Clinical Feedback. The SASSI Institute.

[18] Tiburcio, N. J., Baker, S. L., and Kimmell, K. S. (2020). The Adolescent SASSI-A3 User Guide and Manual. The SASSI Institute.

[19] Mojtabai, R., and Olfson, M. (2020). National trends in mental health care for US adolescents. Jama Psychiatry, 77 (7), 703-714.

[20] National Institute on Drug Abuse. (2007). Addiction and Co-Occurring Mental Disorders.

[21] Pedersen, E. R., Miles, J. N. V., Osilla, K. C., Ewing, B. A., Hunter, S. B., and D'Amico, E. J. (2015). The effects of mental health symptoms and marijuana expectancies on marijuana use and consequences among at-risk adolescents. Journal of Drug Issues, 45 (2), 151-165.

[22] Kuepper, R., Van Os, J., Lieb, R., Wittchen, H., Hofler, M., and Henquet, C. (2011). Continued cannabis use and risk of incidence and persistence of psychotic symptoms: 10 year follow-up cohort study. British Medical Journal, 342, d738.

[23] Piazza, N. J. (1996). Dual diagnosis and adolescent psychiatric inpatients. Substance Use \& Misuse, 31 (2), 215-223.

[24] Child Mind Institute \& Center on Addiction. (2019). Substance Use + Mental Health in Teens and Young Adults: Your Guide to Recognizing and Addressing Co-occurring Disorders.

[25] Hammond, C. J., and Pravesh, S. (2017). Treatment strategies for substance use disorders in adolescents: A clinical review. Psychiatric Times.

[26] Chi, F. W., Sterling, S., Campbell, C. I., and Weisner, C. (2013) Twelve-Step Participation and Outcomes over Seven Years among Adolescent Substance Use Patients with and without Psychiatric Comorbidity. Substance Abuse, 34 (1), 33-42. 